\title{
Migräne ohne Aura Prophylaxe bei häufigen Attacken
}

Patientin J.F, weiblich, geboren 29.06.1976. Die Patientin befindet sich seit April 2004 in meiner ambulanten Behandlung.

\section{Migräneanamnese}

Die Patientin, derzeit 29 Jahre alt, leidet seit dem 22. Lebensjahr unter wiederkehrenden Kopfschmerzattacken. Die Diagnose einer Migräne sei bereits vor Jahren vom Hausarzt gestellt worden. Die Frequenz habe nach ihrer Erinnerung in den letzten Jahren zwei Attacken pro Monat betragen. Die Heftigkeit wird als äußerst intensiv geschildert, einhergehend mit einer völligen Aufhebung der üblichen Alltagsaktivitäten und erforderlicher Bettruhe. Der Charakter ist pochend. Bei physischer Aktivität verschlimmern sich die Schmerzen. Die Cephalgien treten einseitig (mal links, mal rechts) auf. Begleitend kommt es zu als sehr unangenehm erlebten vegetativen Symptomen wie Übelkeit (selten auch Erbrechen), Licht-, Geräusch- und Geruchsüberempfindlichkeit. Insgesamt kann zweifellos nach IHS-Kriterien eine Migräne ohne Aura diagnostiziert werden.

Reproduzierbare Trigger der Schmerzattacken hat Frau F. nicht identifizieren können. Bislang wurde zur Attackenbehandlung Optalidon $\mathrm{N}$ eingesetzt, mit allerdings unzureichendem Erfolg. Eine vorbeugende Behandlung war bislang noch nicht in Erwägung gezogen worden.

\section{Weitere Eigenanamnese}

Keine relevanten Vor- oder Begleiterkrankungen. Es besteht keine Dauermedikation.

\section{Untersuchungsbefund}

Der körperliche Befund war bei der adipösen Frau (Größe $164 \mathrm{~cm}$, Gewicht 80 kg, BMI 30) sowohl in allgemein-klinischer als auch in neurologischer Hinsicht unauffällig.

\section{Weitere Diagnostik}

Ein aktuelles EEG zeigte keine Auffälligkeiten. Auf darüber hinaus- gehende, etwa bildgebende Diagnostik konnte verzichtet werden.

\section{Therapeutisches Vorgehen}

Die Patientin wurde zunächst ausführlich über die Diagnose und die medikamentösen und nicht-medikamentösen therapeutischen Optionen informiert. Es wurde das Führen eines Kopfschmerzkalenders verabredet, um über die folgenden Wochen prospektiv Frequenz und Intensität der Kopfschmerzen zu dokumentieren. Aktuell erschien es sinnvoll, zunächst die Attackenbehandlung $\mathrm{zu}$ optimieren. Hierzu wurde der Einsatz eines Triptans vorgesehen und Rizatriptan (Maxalt ${ }^{\circledR}$ lingua $10 \mathrm{mg}$ ) ausgewählt.

\section{Verlauf}

Die Auswertung des Kopfschmerzkalenders über einige Monate zeigte eine höhere als von Frau F. zunächst angenommene Migränefrequenz mit durchschnittlich fünf jeweils eintägigen Attacken pro Monat. Die Einnahme von Rizatriptan $10 \mathrm{mg}$ erwies sich als gut und rasch wirksam, durchschnittlich wurden pro Monat dementsprechend fünf Einzeldosen Rizatriptan eingenommen bei problemloser Verträglichkeit. Trotzdem fühlte sich Frau F. durch die häufigen, unvorhersehbar auftretenden Kopfschmerzepisoden in ihrem Allgemeinbefinden und in ihrer Lebensqualität erheblich beeinträchtigt, sodass ihr die Möglichkeiten der medikamentösen Prophylaxe detailliert erläutert wurden. Angesichts des Risikos einer Gewichtszunahme unter den zur Prävention zugelassenen Substanzen entschied sich Frau F. für Topiramat (TOPAMAX ${ }^{\circledR}$ MIGRÄNE). Sie erhält die Substanz seit September 2004.

\section{Verlauf unter}

\section{Topiramat-Therapie}

Die Eindosierung erfolgte, ausgehend von einer abendlichen Einmaldosis von $25 \mathrm{mg}$, mit einer wochenweisen Steigerung um 25 mg bis zu einer Enddosis von $100 \mathrm{mg}$ täglich, verteilt auf zwei Dosen. Innerhalb des ersten Monats der Einnahme nahm die Häufigkeit der Attacken auf zwei Episoden pro Monat ab. Hinsichtlich der Verträglichkeit berichtete Frau F. über gering ausgeprägte Parästhesien der Hände und Schmeckstörungen, die von ihr ohne weitere Intervention toleriert wurden.

In den nächsten Monaten stellte sich eine Migränefrequenz von 3 Tagen pro Monat dar, gleichzeitig nahm aber auch die Schmerzintensität $a b$, sodass der Verbrauch an Akutmedikamenten reduziert werden konnte. Die Nebenwirkungen klangen innerhalb einiger Monate ab.

\section{Zusammenfassung}

Topiramat hat sich bei dieser Patientin als eine wirksame, sichere und gut verträgliche Substanz in der prophylaktischen Therapie der Migräne erwiesen. Hervorzuheben ist, dass der therapeutische Effekt auch langfristig erhalten bleibt.

\section{Anschrift des Verfassers}

Dr. Borries Kukowski

Groner-Tor-Str. 5

37073 Göttingen 